\title{
Natural Convection in Inclined Porous Square Enclosure
}

\author{
Abdennacer Belazizia ${ }^{1}$, Smail Benissaad ${ }^{2}$ and Said Abboudi $^{3}$ \\ ${ }^{1}$ Université Larbi Tebessi Tebessa Algérie \\ ${ }^{2}$ Laboratoire d'energétique appliquée et de la pollution. Université Mentouri Constantine1 Algérie \\ ${ }^{3}$ Laboratoire ICB Equipe COMM Université UTBM Belfort France
}

\begin{abstract}
Steady, laminar, natural convection flow in porous square enclosure with inclination angle is considered. The enclosure is filled with air and subjected to horizontal temperature gradient. DarcyBrinkman-Forchheimer model is considered. Finite volume method is used to solve the dimensionless governing equations. The physical problem depends on five parameters: Rayleigh number $\left(R a=10^{3}-10^{6}\right)$, Prandtl number $(P r=0.71)$, Darcy number $(D a=0.01)$, inclination angle $\varphi=\left(0^{\circ}-227^{\circ}\right)$, porosity of the medium $(\varepsilon=0.7)$ and the aspect ratio of the enclosure $(A=1)$. The main focus of the study is on examining the effect of Rayleigh number on fluid flow and heat transfer rates. The effect of inclination angle is also considered. The results including streamlines, isotherm patterns, flow velocity and the average Nusselt number for different values of $R a$ and $\varphi$. The obtained results show that the increase of $R a$ leads to enhance heat transfer rate. The fluid particles move with greater velocity for higher thermal Rayleigh number. Also $\varphi$ affects the fluid motion and heat transfer in the enclosure. Velocity and heat transfer are more important when $\varphi$ takes the value $\left(30^{\circ}\right)$.
\end{abstract}

\section{Introduction}

Natural convection in a fluid saturated porous medium occurs in a wide variety of applications such as heat exchangers, solar power collectors, grain storage, energy efficient drying process, etc. Various modes of convection are possible depending on how temperature and concentration gradients are oriented relative to each other as well as to gravity.

Mohamad et al.[1] investigated numerically doublediffusive natural convection in a horizontal enclosure filled with saturated porous medium. Brinkman extension of Darcy model is adopted. The objective of the work is to understand the physics of the flow and to identify the flow regimes for thermal and solutal dominated flows.

Younsi et al.[2] studied numerically the two-dimensional double diffusive opposing flow in a porous cavity. The Darcy equation including Brinkman-Forchheimer terms to account for viscous and inertia effects, is used for momentum equation. It is shown that the main effect of the porous medium is to reduce the heat and mass transfer as well as the flow field when the permeability is reduced.

Wang et al.[3] analyzed numerically natural convection of fluid in an inclined enclosure filled with porous medium in a strong magnetic field. The BrinkmanForchheimer extended Darcy model is used. The results show that both the magnetic force and the inclination angle have significant effect on the flow field and heat transfer in porous medium.

Sathiyamoorthy et al. [4] reported numerically natural convection flow in a square cavity filled with a porous matrix. Darcy-Forchheimer model without the inertia term is used to simulate the momentum transfer in the enclosure.

In this work we present a numerical study of laminar natural convection in a porous inclined square enclosure. This last is filled with air and submitted to horizontal temperature gradient. Darcy equation including Brinkman-Forchheimer terms is considered to account viscous and inertia effects. The main focus is on examining the effect of Rayleigh number on fluid flow and heat transfer in the enclosure. The effect of inclination angle is also considered. The rate of heat transfer in the enclosure is measured in terms of the average Nusselt number.

\section{Problem Geometry}

The geometry of the problem is shown in Fig.1. The heated vertical left side wall and cooled vertical right side wall of the enclosure are maintained at two different but uniform temperatures: $\left(T_{\max }>T_{\min }\right)$. The remaining boundaries of the enclosure are impermeable and thermally insulated.

\section{Basic Equation}

The flow in the enclosure is assumed to be twodimensional. All fluid properties are constant. The fluid is considered to be incompressible and Newtonien. The Boussinesq approximation is applied $\rho(T)=\rho_{0}\left[1-\beta_{t}\left(T-T_{0}\right)\right]$. Viscous dissipation, heat generation, and radiation are neglected. The governing

\footnotetext{
* Corresponding author: nacer.belazizia@gmail.com
} 
non-dimensional mass, momentum and energy equations are as follows, respectively;

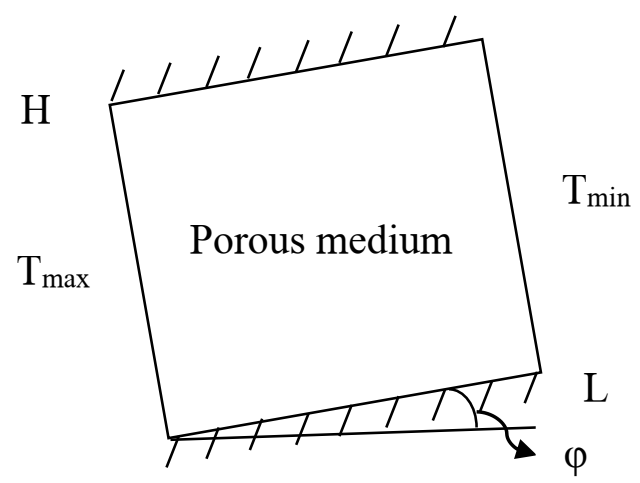

Fig 1. Physical configuration

- at $t=0: U=V=0 ; \theta=0 ; 0 \leq X \leq 1,0 \leq Y \leq 1$

- for $t>0$;

$$
\begin{gathered}
\frac{\partial U}{\partial X}+\frac{\partial V}{\partial Y}=0 \\
\frac{\partial U}{\partial t}+U \frac{\partial U}{\partial X}+V \frac{\partial U}{\partial Y}=-\frac{\partial P}{\partial X}+P_{r} \nabla^{2} U- \\
{\left[\frac{\operatorname{Pr} \cdot \varepsilon}{D a}+\frac{\varepsilon^{2} \cdot C f}{\sqrt{D a}}|\vec{V}|\right] U+\operatorname{Pr} \cdot \operatorname{Ra} \cdot \varepsilon \cdot \theta \cdot \sin \varphi} \\
\frac{\partial V}{\partial t}+U \frac{\partial V}{\partial X}+V \frac{\partial V}{\partial Y}=-\frac{\partial P}{\partial Y}+P_{r} \nabla^{2} V- \\
{\left[\frac{\operatorname{Pr} \cdot \varepsilon}{D a}+\frac{\varepsilon^{2} \cdot C f}{\sqrt{D a}}|\vec{V}|\right] V+\operatorname{Pr} \cdot \operatorname{Ra} \cdot \varepsilon \cdot \theta \cdot \cos \varphi} \\
\frac{\partial \theta}{\partial t}+U \frac{\partial \theta}{\partial X}+V \frac{\partial \theta}{\partial Y}=\nabla^{2} \theta
\end{gathered}
$$

The boundary conditions in the dimensionless form are:

$$
\begin{gathered}
X=0: U=V=0, \text { for } 0 \leq Y \leq 1 \\
X=0: \theta=1, \text { for } \quad 0 \leq Y \leq 1 \\
X=1: U=V=0, \theta=0,0 \leq Y \leq 1 \\
Y=0: U=V=0, \frac{\partial \theta}{\partial X}=0, \text { for } 0 \leq X \leq 1 \\
Y=1: \quad U=V=\frac{\partial \theta}{\partial Y}=0, \text { for } 0 \leq X \leq 1
\end{gathered}
$$

The average Nusselt number is:
Left wall: $\quad \overline{N u}=-\int_{0}^{1} \frac{\partial \theta}{\partial X} d y$;

Right wall: $\quad \overline{N u}=-\int_{0}^{1} \frac{\partial \theta}{\partial X} d y$

\section{Numerical Method}

The governing equations (1) to (4) associated with the boundary conditions (5) are solved numerically using the finite volume method described by Patankar [6]. A uniform mesh is used in $X$ and $Y$ directions Fig2. A hybrid scheme and first order implicit temporally discretisation are used. Because of the nonlinearity of the momentum equations, the velocity pressure coupling, and the coupling between the flow and the energy equation, an iterative solution is necessary. The SIMPLER algorithm and Tri-Diagonal Matrix algorithm iteration procedure [6] are used to solve the algebraic equations. The iteration process is terminated under the following conditions (eqs 7 and 8):

$$
\sum_{i, j}\left|\phi_{i, j}^{n}-\phi_{i, j}^{n-1}\right| / \sum_{i, j}\left|\phi_{i, j}^{n}\right| \leq 10^{-5}
$$

Where $\phi$ represent: $U, V$ and $\theta$; n denotes the iteration step.

$$
\left.\overline{N u}\right|_{X=0}=\left.\overline{N u}\right|_{X=1}
$$

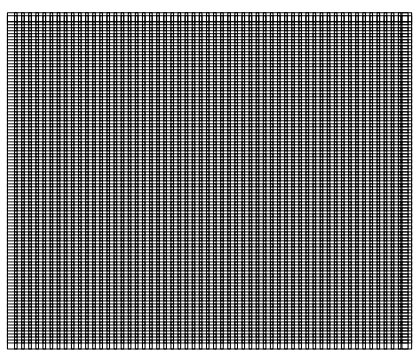

Fig 2. Uniform mesh in $X$ and $Y$ directions

In order to obtain a precise results a $(60 \times 60)$ grid was selected and used in all the computations Fig 3. A good agreement between the obtained results and thows reported in literature [5] are observed (Tab.1). 


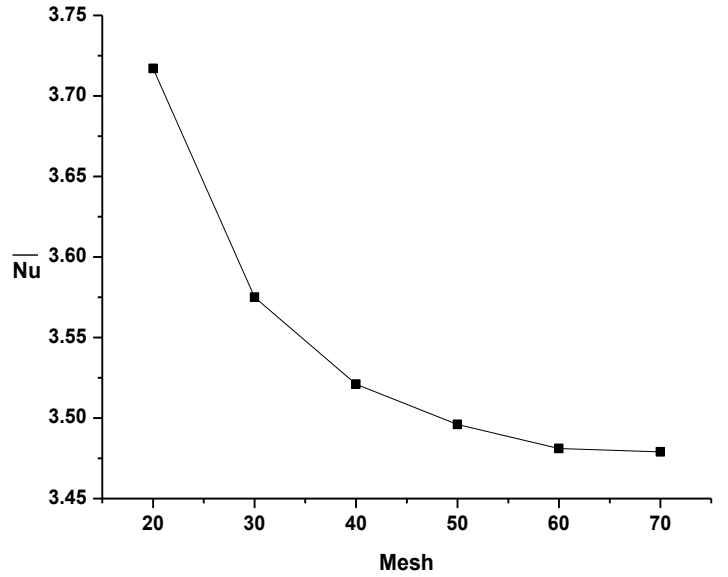

Fig 3. Average Nusselt number for different grid sizes.

Table 1 Average Nusselt number for different Raileigh number. $\operatorname{Pr}=0.71$

\begin{tabular}{|c|c|c|}
\hline $\boldsymbol{R} \boldsymbol{a}$ & present study & [5] \\
\hline $10^{3}$ & 1.118 & 1.116 \\
\hline $10^{4}$ & 2.251 & 2.238 \\
\hline $10^{5}$ & 4.567 & 4.509 \\
\hline
\end{tabular}

\section{Results and Discussions}

\subsection{Effet of Rayleigh number}

In the absence of inclination angle $\left(\varphi=0^{\circ}\right)$, Fig. 4 shows the effect of Rayleigh number on fluid motion inside the enclosure. A single cell rotating in clockwise direction appears inside the enclosure. A weak convection is observed for low Rayleigh number $\left(R a=10^{3}\right)$. While for the remaining cases streamlines cover the entire enclosure and the centre of each cell is elongated and two secondary vertices appear inside it $\left(R a=10^{6}\right)$. In addition by moving from $R a=10^{3}$ to $10^{6}$ the maximum values of velocity (Tab2) is more important which means natural convection is strength in this case.
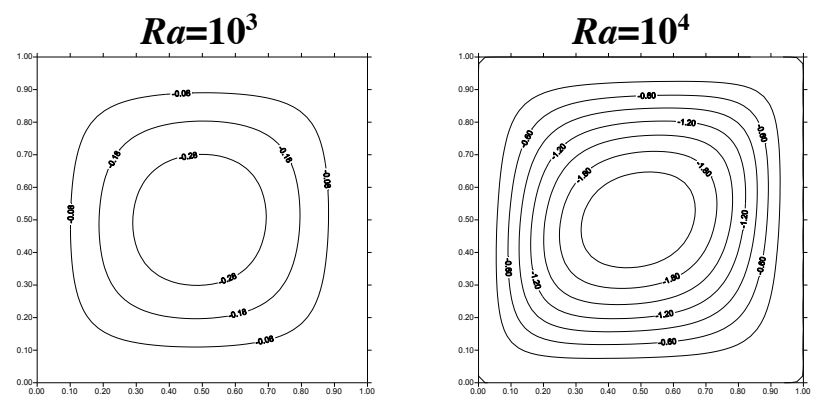
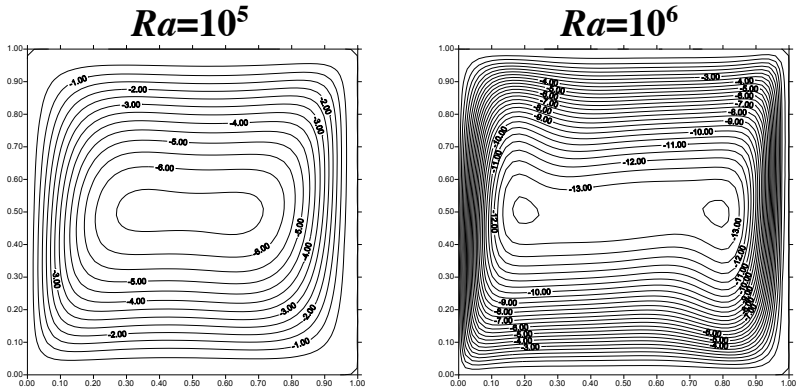

Fig 4. Steady state of streamlines: for different values of Rayleigh number. $\varphi=0^{\circ}$.

Table 2 Maximum velocity for different values of Rayleigh number. $\varphi=0^{\circ}, D a=0.01$.

\begin{tabular}{|c|c|c|c|c|}
\hline$R a$ & $10^{3}$ & $10^{4}$ & $10^{5}$ & $10^{6}$ \\
\hline$V_{\max }$ & 1.175 & 8.882 & 41.143 & 144.61 \\
\hline
\end{tabular}

Also it is observed that the fluid particles move with greater velocity for higher Rayleigh number, as mentioned in Fig.5 showing the effect of $R a$ on midheight horizontal velocity profiles. The velocity peaks especially near the horizontal walls are more important for high $R a$.

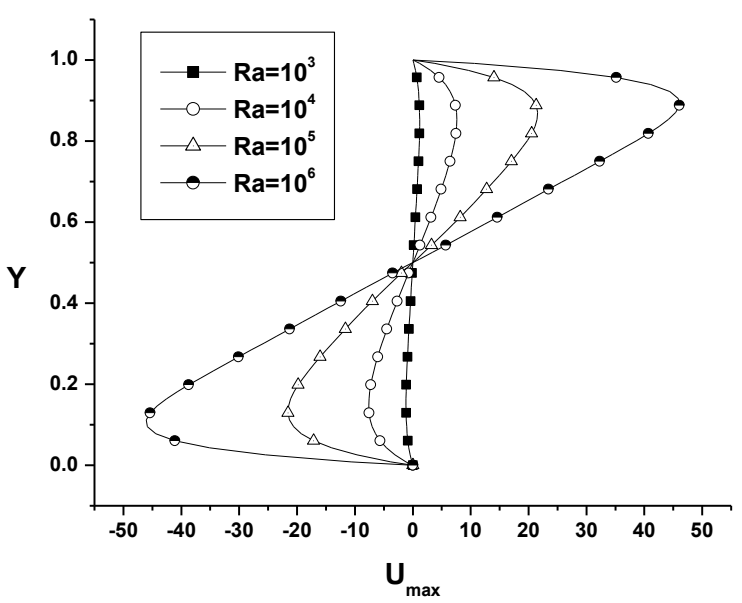

Fig 5. Axial velocity $U$ at $X=0.5$ for different values of Rayleigh number. $\varphi=0^{\circ}, D a=0.01$.

The effect of Rayleigh number on thermal field is illustrated in Fig.6. The fluid rises along the hot wall and falls along the right cold wall. Thermal gradients are very important and isotherms are crowded around the vertical walls for high Rayleigh number. For low $R a$ the isotherms shown in Fig.6 are almost parallel to the vertical walls, indicating that most of the energy transfer is by heat conduction. For high $\left(R a=10^{6}\right)$ there is a temperature stratification in the vertical direction and the thermal boundary layer is well established along the side 
walls indicating the dominance of convection heat transfer mechanism.

The rate of heat transfer across the cavity is obtained by evaluating the average Nusselt number at the cavity walls. Fig.7 presents the effect of Rayleigh number on $\overline{\mathrm{Nu}}:$ It is clear from this figure that the average Nusselt number is increasing with Rayleigh number. For low $R a$ $\left(R a=10^{3}\right)$, heat transfer is dominated by diffusion mode $\overline{N u}=1$.
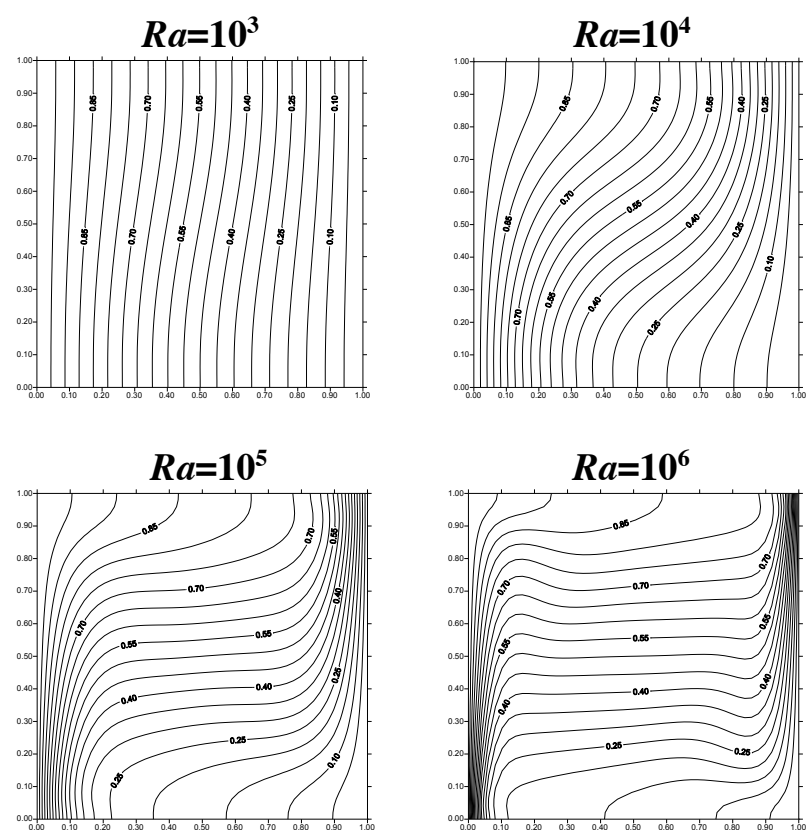

Fig 6. Steady state of isotherms for different values of thermal Rayleigh number.

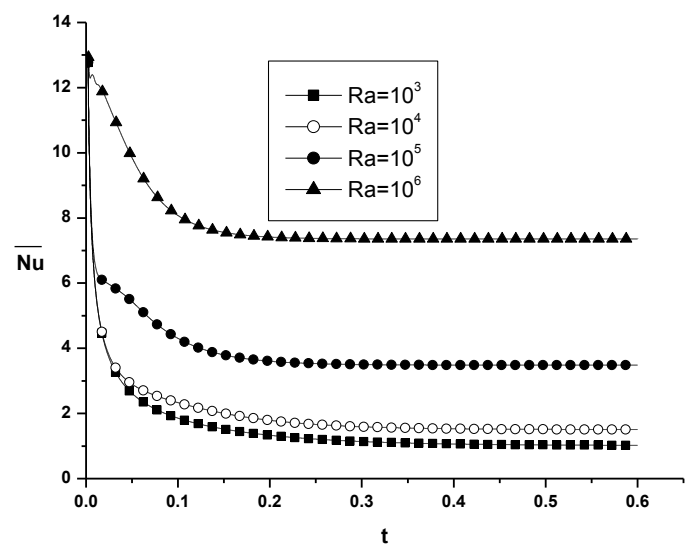

Fig 7. Average Nusselt number versus time for different Rayleigh number.

\subsection{Effect of inclinition angle $\varphi$}

In order to show the effect of the inclination angle of the porous enclosure on natural convection, we have selected the following control parameter: $\varphi=0^{\circ}$ to $270^{\circ}$, $D=0.01$ and $R a=10^{5}$. Figs 8, 9 and 10 show the effect of $\varphi$ on fluid motion, isotherm lines and heat transfer rates. The maximum velocity in the flow and the average Nusselt number are the greatest in the case $\varphi=30^{\circ}$. They are less important in the case $\varphi=270^{\circ}$. For $\varphi=90^{\circ}$ and $\varphi=270^{\circ}$ the enclosure is heated respectively in the lower horizontal wall and the top horizontal wall. In the first case the flow structure is bicellular, where as in the second case we note that no flow in the enclosure and the fluid is stratified in the vertical direction.
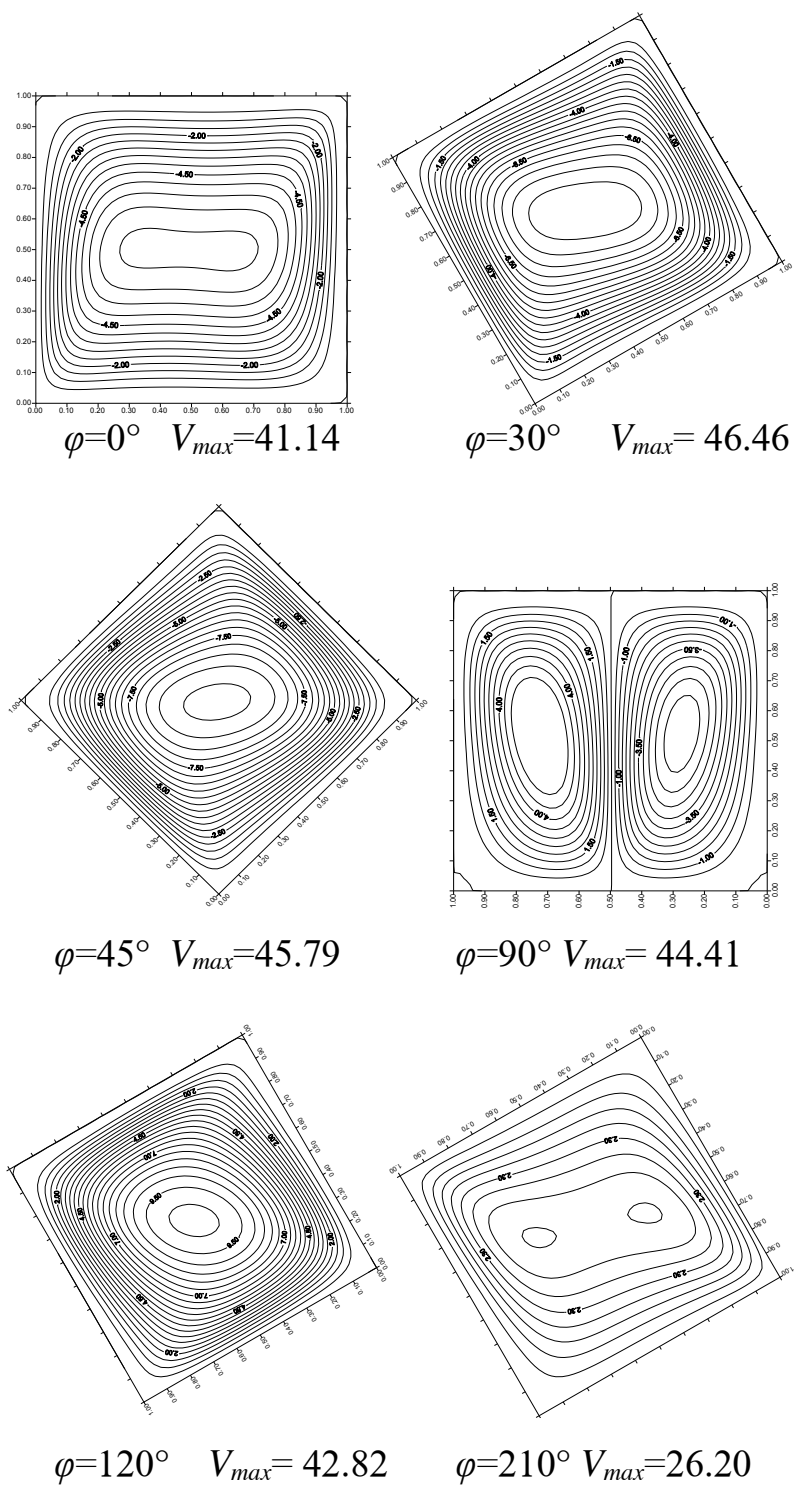

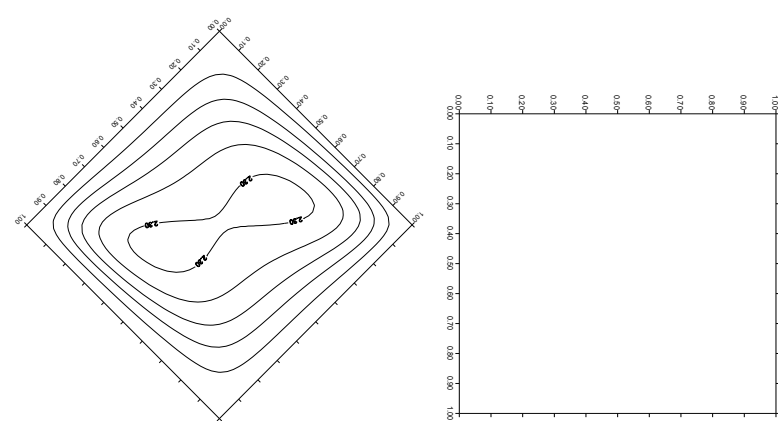

$\varphi=225^{\circ} \quad V_{\max }=16.62$

$$
\varphi=270^{\circ} \quad V_{\max }=0.0
$$

Fig 8. Steady state of streamlines: for different values of $\varphi$.
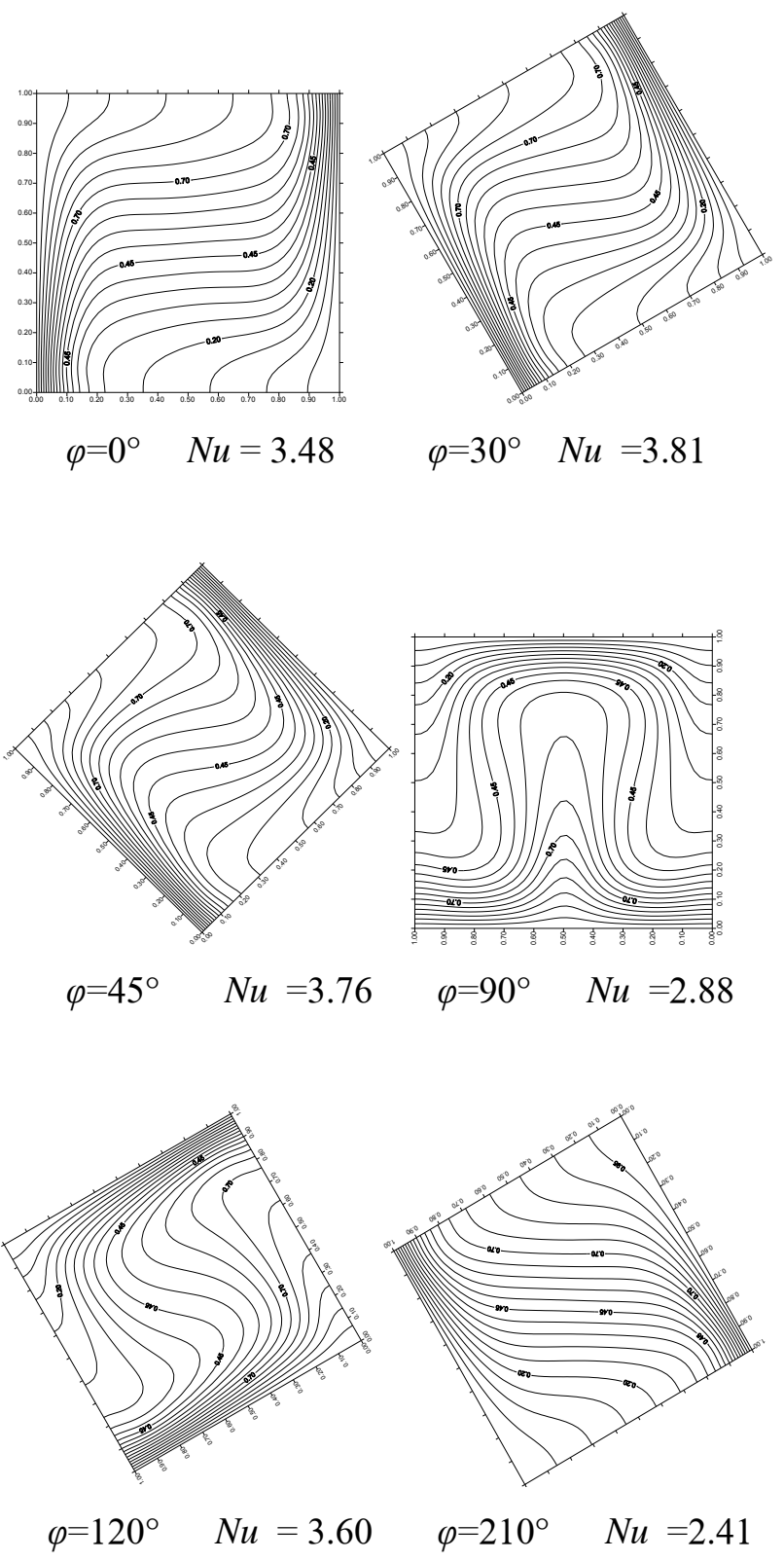

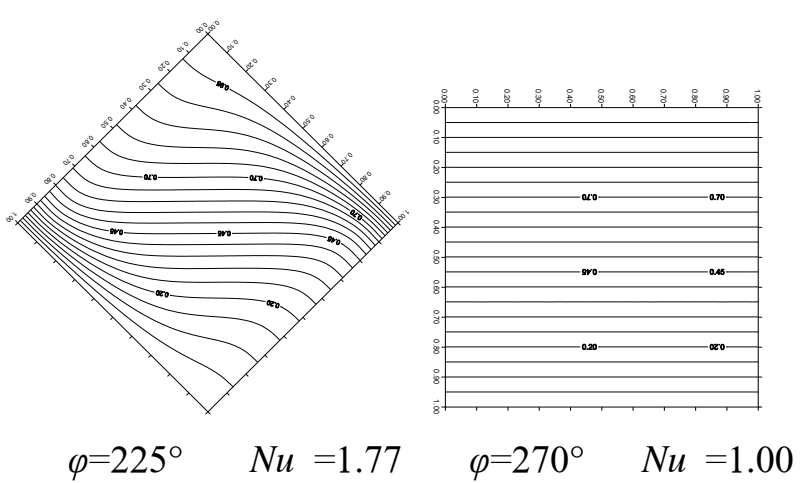

Fig 9. Régime stationnaire des isothermes pour différentes valeurs de $\varphi$.

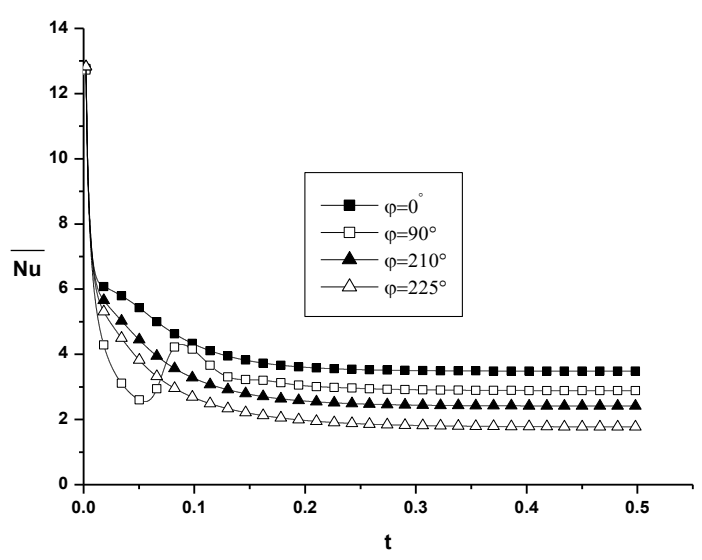

Fig 10. Average Nusselt number versus time for different inclination angle.

\section{Conclusions}

A numerical study of natural convection was employed to analyze the flow and heat transfer in a square porous enclosure. Rayleigh number and inclination angle have a noticeable effect on fluid motion and heat transfer rate in the enclosure. The following conclusions are summarized:

- In case $(\varphi=0)$ the increase of Rayleigh number, leads to increase flow convection and heat transfer rates. For low Rayleigh number heat transfer is dominated by diffusion mode

- In case $(\varphi \neq 0)$ and for high Rayleigh number $\left(R a=10^{5}\right)$, the angle $\varphi=30^{\circ}$ gives the highest velocity flow and the highest heat transfer rate. In contrast, for the angle $\varphi=270^{\circ}$, isotherms are parallel to the active walls. This indicates the stratification of the thermal field and heat conduction is dominated in the enclosure.

\section{References}

1. A. Mohamad, and R. Bennacer, Int.J.Ther.Sci. 40, 82-93, (2001). 
2. R. Younsi, A. Harkati, and D. Kalache, Arab.J. Sci and Eng. 27, 2c, (2002).

3. Q.W. Wang, M. Zeng, Z.P. Huang, , G. Wang, and H.Ozoe, Int. J.heat and mass transfer 50, ( 2007)

4. M. Sathiyamoorthy, T. Basak, S. Roy, and I. Pop, Int. J. of heat and mass transfer 50, (2007).

5. G. D. Vahl Davis, Int. J. Num. Mesh. Fluids 3, (1983).

6. S. V. Patankar, McGraw-Hill, Washington, DC. (1980).

\section{Nomenclature}

A aspect ratio, $H / L$

Da Darcy number, $K / H^{2}$

$\mathrm{K} \quad$ medium permeability.

$\overline{\mathrm{Nu}} \quad$ average Nusselt number.

$P \quad$ dimensionless pressure, $p /(\alpha / H)^{2}$

$\operatorname{Pr} \quad$ Prandtl number of the fluid, $v / \alpha$

$R a \quad$ thermal Rayleigh number, $g \beta_{t} H^{3} \Delta T / v \alpha$

$t \quad$ dimensionless time, $t^{*} /\left(H^{2} / \alpha\right)$

$U, V \quad$ dimensionless velocity components, $u /(\alpha / H)$,

$v /(\alpha / H)$

$X, Y \quad$ non-dimensional cartesian coordinates, $x / H$, $y / H$

\section{Greek symbols}

$\begin{array}{ll}\varepsilon & \text { medium porosity } \\ \varphi & \text { inclnation angle } \\ \theta & \text { non-dimensional temperature, }\left(T-T_{\min }\right) / \Delta T \\ \psi & \text { non-dimensional stream function, } U=\partial \psi / \partial Y \\ \Delta T & \text { temperature difference, }\left(T_{\max }-T_{\min }\right)\end{array}$

